\title{
La profesión habitual una referencia a modificar en la valoración de la incapacidad laboral
}

\author{
The usual profession a reference to change in the assessment of incapacity
}

\author{
José Manuel Vicente Pardo \\ Unidad Médica Equipo Valoración Incapacidades Gipuzkoa. INSS. España \\ Recibido: 28-01-15 \\ Aceptado: 05-02-15

\section{Correspondencia} \\ José Manuel Vicente Pardo \\ Pescadores Gran Sol s/n \\ San Sebastián 20011 , Gipuzkoa. España. \\ Correo electrónico: jose-manuel.vicente@seg-social.es
}

Resumen

En nuestro sistema de seguridad social la profesión o el carácter profesional o el criterio de profesionalidad son determinantes a la hora de establecer el grado de incapacidad, si bien en un repaso exhaustivo al concepto, la legislación y la práctica diaria, se entiende es un término inadecuado para la referencia actualizada y en consonancia con los criterios de medicina evaluadora sobre valoración incapacitante laboral en nuestro sistema de seguridad social.

Conclusión: Las referencias a profesión habitual son obsoletas e imprecisas. El concepto de PROFESIÓN HABITUAL debe revisarse, tanto en su valoración médico-laboral como de referencia normativa-legal de cara a la valoración de la capacidad o incapacidad laboral en el sistema de seguridad social. La profesión habitual es difícil de definir en la actualidad por los continuos cambios en los procesos productivos, la especialización creciente, la automatización de muchos de ellos y los nuevos trabajos en el entorno telemático. Así como por la tendencia a la temporalidad y precariedad en el desempeño de una "profesión». Sería preferible referirse cuando menos a Grupos Profesionales con similares requerimientos de capacidades, parecidas condiciones de trabajo y riesgos presentes.

El propio término de profesión es confuso e impreciso pues puede referenciar tanto profesión como ocupación, puesto de trabajo, tarea o actividad, en un medio laboral cambiante muy diversificado y especializado y en continuo cambio y con aparición de profesiones nuevas o renovación de las requisitorias de las antiguas y con un índice de rotación por múltiples trabajos que será algo muy habitual en la población trabajadora a lo largo de su vida laboral. Es urgente y necesario establecer protocolos que valoren las concretas, específicas y reales, capacidades requeridas por el trabajo, sus condiciones y riesgos presentes y su formación, de un trabajo concreto, más allá de unas referencias genéricas a profesiones.

Med Segur Trab (Internet) 2015; 61 (238) 68-77

Palabras clave: profesión, valoración incapacidad laboral, aptitud, capacidades y requerimientos del trabajo. 


\section{Abstract}

In our system of social security profession or professional nature or the criterion of professionalism are determining factors in establishing the degree of disability, although an exhaustive to the concept overview, legislation and daily practice, the term is inadequate term for the date and consistent with the criteria for evaluating medical assessment on labor disabling our social security system reference.

Conclusion: References to normal profession are outdated and inaccurate. The concept of USUAL OCCUPATION be reviewed in both its medical-employment assessment and normative-legal point of comparison for assessing the capacity or incapacity in the social security system. The usual occupation is difficult to define today by continuous changes in production processes, increasing specialization, automation and many new jobs in the telematics environment, and by the tendency to temporality and precariousness in the performance of a "profession". It would be preferable to refer at least to Professional Groups with similar capacity requirements, similar working conditions and hazards present. The term profession itself is confusing and imprecise as it can reference both profession and occupation, job, task or activity in a changing work environment very diversified and specialized and continuous change and emergence of new professions or renewal of the requisitions of old and with a turnover of multiple jobs that will be very common in the working population throughout their working lives. It is urgent and necessary to establish protocols that assess the concrete, specific and actual skills required for the job, conditions and risks involved and their training for a particular job, beyond a generic references professions.

Med Segur Trab (Internet) 2015; 61 (238) 68-77

Keywords: profession, valuation disability, aptitude, fitness to works, kills and job requirements. 


\section{PROFESIÓN HABITUAL. CONCEPTO}

En nuestro sistema de seguridad social la profesión o el carácter profesional o el criterio de profesionalidad son determinantes a la hora de establecer el grado de incapacidad, baste una referencia como la que haremos a continuación cuando hablemos de los grados de incapacidad para ver como la profesión habitual en su denominación la referimos textualmente en la incapacidad parcial o total y más allá en el extremo absoluto para toda profesión u oficio.

No deja de ser ilustrativo que esta acepción o identificación con la profesión no se haga, ni tampoco la del término de "incapacidad" cuando nos referimos a las Lesiones Permanentes No Invalidantes (Baremos) o a la Gran Invalidez. Es decir no importa el trabajo o la profesión como determinante para establecer su consideración.

Y otra particularidad a estas indemnizaciones por daños derivados del trabajo o contingencia profesional caso de los «baremos» o lesiones permanentes no invalidantes o en el supuesto de la "compensación» o complemento añadido a la pensión de incapacidad por la necesidad de tercera persona que es lo que es la gran Invalidez, como vemos respetan el término de invalidez.

El término de invalidez que se sustituyó por el de incapacidad, actualizando la expresión, de conformidad con art. 8.5 de la L. 24/1997, de 15 de julio, de Consolidación y Racionalización del Sistema de Seguridad Social.

Pero:

- Se conserva el término en invalidez en la Gran Invalidez y en las Lesiones Permanentes No Invalidantes o Baremos por Accidente de Trabajo o de Enfermedad Profesional; ya que en ambas situaciones no se guarda la relación obligada con el trabajo que se da para la Incapacidad Permanente Parcial para la profesión habitual, la Incapacidad Permanente Total para la profesión habitual o la Incapacidad Permanente Absoluta para toda profesión.

- Pues da igual cual sea, así en el primer caso Gran Invalidez (GI) el complemento de GI se otorga por la necesidad que tiene el trabajador necesitar de tercera persona para sus actividades de autocuidado o de la vida diaria y en el segundo caso el de los Baremos, es un listado de baremos que indemnizan por el daño causado independientemente de cuál sea el trabajo.

Así pues en el sistema de calificación de la incapacidad (contributiva) en seguridad social, es un sistema de evaluación de daños producidos por la actualización del riesgo de carácter profesional.

El criterio de "profesionalidad" atiende más bien a la capacidad residual genérica del trabajador, y no a la específica o concreta, que con el paso del tiempo o en su proyección a futuro tiende a hacerse irrelevante.

Artículo 137 Ley General Seguridad Social. Grados de Incapacidad.

1. La invalidez permanente, cualquiera que sea su causa determinante, se clasificará con arreglo a los siguientes grados:

a) Incapacidad permanente parcial para la profesión habitual.

b) Incapacidad permanente total para la profesión habitual.

c) Incapacidad permanente absoluta para todo trabajo.

d) Gran invalidez.

2. Se entenderá por PROFESIÓN HABITUAL,

- En caso de accidente, sea o no de trabajo, la desempeñada normalmente por el trabajador al tiempo de sufrirlo. 
- En caso de enfermedad común o profesional, aquélla a la que el trabajador dedicaba su actividad fundamental durante el período de tiempo, anterior a la iniciación de la incapacidad, que reglamentariamente se determine.

3. Se entenderá por incapacidad permanente parcial para la profesión habitual la que, sin alcanzar el grado de total, ocasione al trabajador una disminución no inferior al 33 por 100 en su rendimiento normal para dicha profesión, sin impedirle la realización de las tareas fundamentales de la misma.

4. Se entenderá por incapacidad permanente total para la profesión habitual la que inhabilite al trabajador para la realización de todas o de las fundamentales tareas de dicha profesión, siempre que pueda dedicarse a otra distinta.

5. Se entenderá por incapacidad permanente absoluta para todo trabajo la que inbabilite por completo al trabajador para toda profesión u oficio.

6. Se entenderá por gran invalidez la situación del trabajador afecto de incapacidad permanente y que, por consecuencia de pérdidas anatómicas o funcionales, necesite la asistencia de otra persona para los actos más esenciales de la vida, tales como vestirse, desplazarse, comer o análogos.

Procediendo la declaración de la incapacidad permanente total para la profesión habitual cuando la situación física o psíquica del trabajador le inhabilita para la realización de todas o de las fundamentales tareas de su profesión habitual.

Procediendo la declaración de la incapacidad permanente parcial para la profesión habitual cuando al trabajador mantenga una disminución no inferior al 33 por 100 en su rendimiento normal para dicha profesión habitual, sin impedirle la realización de las tareas fundamentales de la misma.

El concepto de profesión habitual por tanto resulta obligado en la determinación de la incapacidad total y parcial.

Concepto profesional o laboral en la determinación de la incapacidad que es más genérico para la incapacidad absoluta, pues se habla no de profesión habitual sino de todo trabajo, toda profesión u oficio.

Hay dos tipos de caracteres que conceptúan la incapacidad permanente:

- Unos referidos a lesiones que tienen que ser clínicamente objetivables, que deben ser definitivas o permanentes en el sentido de no ser posiblemente mejorables con tratamiento médico.

- Y otros referidos a la incidencia de las lesiones sobre la capacidad laboral del individuo.

Utilizando como "parámetro" en su gradación, bien la profesión habitual o bien la referencia a cualquier profesión u oficio. Conociendo que no es un gradación porcentual como otros sistemas o protocolos de valoración del daño.

Añadiendo dos subgrupos de prestación por incapacidad permanente (pues los baremos no dejan de ser una indemnización a tanto alzado es decir por una única vez):

- La Incapacidad Total cualificada, para mayores de 55 años que siendo perceptores o se les conceda el grado de incapacidad total superen esa edad y no tengan trabajo, y en razón a la especial dificultad que pueden tener para obtener empleo en actividad distinta de aquella para al que es declarado incapacitado (art. 139.2 LGSS).

- La Gran Invalidez, ya explicada su no relación con el trabajo o al profesión sino con la necesidad de precisar ayuda para las denominadas de forma genérica actividades básicas de la vida diaria.

Por último, en esta introducción a la incapacidad y la profesión, recordar como en la definición de incapacidad temporal o «baja» (art. 128 LGSS) la situación "de daño» o consecuencia limitante de una enfermedad o lesión (accidente) debe de causar 
impedimento para el trabajo, término "Trabajo» que guarda relación inmediata con el trabajo que está desempeñando ene se momento, más cercano a la tarea o actividad concreta que debe desarrollar en el trabajo que al término más genérico y más amplio y completo de Profesión u Oficio. Bien entendido que en padecimientos graves o extensivos o de especial intensidad, aún en este caso de la incapacidad temporal, puede ser irrelevante el trabajo pues se está impedido para cualquiera aún de forma temporal o transitoria. Manteniendo el rigor en la constatación de la enfermedad del trabajador y de su incidencia en la capacidad para realizar su trabajo.

Pero conviene no perder esta perspectiva de impedido para el trabajo (que se está desempeñando) pues pueden existir supuestos en los que las limitaciones derivadas de un proceso no supongan una pérdida o impedimento apara trabajar pues no son necesarias para el desarrollo del trabajo pues no son requeridas por este.

Supongamos una lesión por un esguince leve de tobillo en un administrativo o en un encofrador, que puede no ser causa de baja en el primer caso y si serlo para el segundo trabajo.

Así por tanto el término de profesión va a condicionar las incapacidades permanentes y el de trabajo las temporales.

\section{PROFESIÓN SÍ, PERO ¿POR QUÉ ES TAN NECESARIO CONOCER SUS CARACTERÍSTICAS?}

- Porque Trabajos diferentes, tienen distintas exigencias.

- Porque las Lesiones son Incapacitantes dependiendo el tipo de trabajo.

- Porque en materia de seguridad social la incapacidad permanente viene referida al término de profesión habitual Incapacidad Permanente Parcial Incapacidad Permanente Total o expandida a toda profesión Incapacidad Permanente Absoluta.

\section{¿Qué debemos entender por profesión?}

- El concepto de profesión engloba todas las funciones propias de la misma, sus labores concretas, las tareas que lo configuran, su formación, sus requerimientos de aptitud funcional, su adscripción normativa y clasificatoria, sus particularidades y condiciones del puesto y área o centro o medio donde se realiza.

- Debe valorarse la profesión, que NO ES siempre concordante con el concreto puesto de trabajo, ni la concreta categoría profesional, valorándose la pérdida de capacidad para su desempeño de manera más importante que la pérdida de capacidad para un puesto o cometido concreto o incluso para una determinada categoría.

- En Incapacidad Temporal es la que realiza en el momento de permanecer en esa situación. Más que profesión hablaríamos de Trabajo, algo más cercano a Puesto concreto, Actividad o Tarea.

- En Incapacidad Permanente el término al uso es PROFESIÓN HABITUAL, o TODA PROFESIÓN, que es algo más cercano a OCUPACIÓN, a OFICIO, que a actividad concreta. Se valora la pérdida de capacidad para el desempeño de la profesión.

- En contingencia común profesión habitual es la desarrollada a lo largo de la vida activa.

- En contingencia profesional (accidente de trabajo o enfermedad profesional) la que desarrollaba en el momento de sufrir el AT o la actividad en la desarrolló la EP. 


\section{CONSIDERACIONES LEGALES O NORMATIVAS}

La Jurisprudencia y La Ley 24/97 orienta el concepto de profesión habitual al grupo profesional, definido en el artículo 22 del Estatuto de los Trabajadores.

La Ley 27/2011 modifica el artículo 141 de la LGSS y orienta dicho concepto de profesión habitual a las funciones laborales objeto de concesión de incapacidad.

- Profesión que ejercía el interesado o del grupo profesional en que aquélla estaba encuadrada.

- Siempre y cuando las funciones no coincidan con aquellas que dieron lugar a la incapacidad permanente total.

\section{LA JURISPRUDENCIA}

La jurisprudencia identifica la profesión habitual con el conjunto de tareas que conformaban la categoría profesional del trabajador (SSTCT de 1 de marzo, de 15 de abril de 1977 y 6 de febrero de 1986).

La sentencia de 17 de enero de 1989 (STS) vino a señalar expresamente que el punto de referencia en la determinación de la incapacidad «laboral» es la labor que «el trabajador está cualificado para realizar y a lo que la empresa le haya destinado o pueda destinarle en movilidad funcional, sin perjuicio de las limitaciones correspondientes a las exigencias de titulación académica o de pertenencia a un grupo profesional.

De este modo, en la calificación de la invalidez, no sólo se han de tener en cuenta las funciones correspondientes a la categoría profesional del presunto incapaz, sino también aquéllas que le sean exigibles dentro de la movilidad funcional ordinaria (art. 39 ET).

Son muchas las resoluciones que han venido después refiriendo y aplicando este criterio y que definen la profesión habitual como el conjunto de tareas exigibles dentro de la movilidad funcional ordinaria.

Concepto de profesión habitual: profesión habitual no es sólo identificable con un concreto puesto de trabajo sino con aquella que el trabajador este cualificado y a la que se le haya destinado o pueda ser destinado en movilidad funcional.

La profesión habitual engloba todas las funciones propias de la misma. No es esencialmente coincidente con una labor específica, o labores concretas que se realicen determinado puesto.

En supuestos de cambio de puesto de adaptación para valorar si es la profesión habitual u otra distinta habrá que estar a valorar no sólo las funciones o trabajos concretos que estuviera realizando antes y las posteriores al cambio, sino todas las que integran objetivamente su profesión, en ocasiones esta puede venir delimitada por la propia categoría profesional o en otras por el grupo profesional según el alcance que en cada caso tenga el ius variandi empresarial de conformidad con la normativa laboral aplicable.

No basta por tanto para determinar si es profesión habitual u otra distinta la realización de funciones diferentes o de cometidos diferentes, sino el que se haya producido de facto una interrupción de una relación laboral y el nacimiento de otra nueva con nuevas dedicaciones profesionales.

Profesión habitual no es tampoco un puesto de confianza, deben de valorarse todas las tareas o las fundamentales que configuran la profesión.

Por tanto ha de utilizarse un criterio más cualitativo que cuantitativo.

Es decir, para apreciar la disminución del rendimiento, habrá que estar más que a las tareas desarrolladas a lo largo de la jornada laboral, a todas aquellas que, desempeñadas o no, corresponden a la correspondiente categoría profesional. 
Por otra parte y esta concepción de profesión habitual para la estimación de situación incapacitante debe de realizarse más que por las lesiones por las limitaciones orgánicas y funcionales que las mismas producen respecto de los requerimientos psicofísicos de la profesión habitual o toda profesión, atendiendo a las capacidades residuales objetivadas, atendiendo las limitaciones funcionales derivadas de los padecimientos sufridos, sin que debe exigirse un verdadero afán de sacrificio por parte del trabajador y una intensa tolerancia por parte del empresario, debiendo de valorarse el poder realizar la profesión habitual con profesionalidad y con unas exigencias mínimas de continuidad, rendimiento y eficacia, y sin que el desempeño de la profesión genere riesgos adicionales superpuestos a los normales para sí o para terceros o comporte un sacrificio continuado en el trabajo cotidiano, en definitiva la imposibilidad de seguir desarrollando su profesión.

Observancia de protección frente al riesgo, que con carácter preventivo personal o de otros conviene siempre valorar.

En cualquier caso y mientras no se modifique la LGSS, el criterio de referencia es, debe ser la profesión habitual y no el concreto puesto de trabajo que se desempeña, de forma que no se atiende al perjuicio inmediato sino a aquel que define, a más largo plazo, la vida profesional, ya que normalmente se desarrolla una sola en la vida, al margen de que puedan irse escalando determinados niveles o categorías dentro de ella (STSJ País Vasco de 20-1-1998).

\section{CONCEPTO DE PROFESIÓN HABITUAL O PROFESIÓN ÚNICA}

La especialización continuada y cada día más específica en el mundo del trabajo, las nuevas tecnologías, la automatización, la robotización, el continuo cambio en el mundo laboral de los procesos, de la industria, de materiales, de los servicios, condicionan cambios en las condiciones de trabajo y en la preparación del trabajador, a lo que debiéramos añadir la movilidad laboral, la temporalidad en el empleo, y la precariedad laboral.

Por todo ello el concepto de profesión habitual como algo continuado, no es útil a día de hoy pues este hecho pertenece a un pasado con historia laboral continua, sin demasiados cambios en las profesiones $u$ oficios nuevos que choca con la modernización en la industria y en general en todos los trabajos con aparición de nuevas profesiones modificación de otras antiguas adaptándose a nuevos procesos, procedimientos, usos de nuevas maquinarias o instrumentos laborales, nuevas tareas, nuevos modalidades de servicios y por tanto precisar de nuevos requerimientos, capacidades y formación para su desempeño. Sin olvidar la precariedad en el empleo que obliga al trabajador a optar por nuevas opciones laborales que se le presenten, para poder subsistir.

Por ello hay que ir hacia grupos profesionales que incluyan o agrupen los mismos por exigir mismos requerimientos para su desarrollo.

\section{CONCEPTO DE PROFESIÓN HABITUAL ORIENTADO AL DE GRUPO PROFE- SIONAL. LA LEY 24/97}

El artículo 8 de la Ley 24/1997 de 15-7, de Consolidación y Racionalización del Sistema de Seguridad Social, vino a dar nueva redacción al art. 137 LGSS, y también a consagrar esta interpretación más amplia cuando dispone en su aportado quinto que «la calificación de la incapacidad permanente en sus distintos grados se determinará en función del porcentaje de reducción de la capacidad de trabajo que reglamentariamente se establezca (...).

A efectos de la determinación del grado de incapacidad, se tendrá en cuenta la incidencia de la reducción de la capacidad en el desarrollo de la profesión que 
ejercía el interesado o del grupo profesional en que aquélla estaba encuadrada, antes de producirse el becho causante de la incapacidad permanente». Se amplía la determinación de la incapacidad porque se hace en referencia a la profesión que ejercía el interesado pero también al grupo profesional en que aquélla estaba encuadrada.

La referencia que la Ley 24/1997 hace al grupo profesional recoge la jurisprudencia citada, que definía la profesión habitual, con las funciones exigibles al trabajador dentro de la movilidad funcional ordinaria (art. 39.1 ET).

El nuevo concepto de profesión habitual por tanto se delimita no sólo a atender al de "la profesión que ejercía el interesado", sino también al concepto de "grupo profesional», término o concepto más amplio y que no está exento de dificultades calificadoras, ya que la constitución de los grupos profesionales no contiene referencia alguna a las profesiones. Pues, esta referencia al grupo profesional, puede ser menos favorable para el trabajador generando supuestos de desprotección si la afectación de la capacidad laboral impide ejercer las tareas de su profesión u oficio pero puede ejercer otras funciones o tareas de distinta profesión encuadrada dentro de su grupo profesional.

La reforma efectuada en el artículo 137.2 LGSS por la Ley 24/1997 identificaba profesión habitual como grupo profesional, recordemos que expresamente contempla que a efectos de la determinación del grado de la incapacidad se tendrá en cuenta la incidencia de la reducción de la capacidad de trabajo en el desarrollo de la profesión que ejercía el interesado o del grupo profesional, en que aquélla estaba encuadrada, antes de producirse el hecho causante de la incapacidad permanente. Desde una interpretación literal del artículo 137.2 LGSS (no vigente).

\section{PROFESIÓN HABITUAL, FUNCIONES LABORALES. LA LEY 27/2011 MODIFI- CA EL ARTÍCULO 141 DE LA LGSS}

La Ley 27/2011 modifica el artículo 141 de la LGSS y orienta dicho concepto de profesión habitual a las funciones laborales, lo que viene a conectar con la propuesta de valorar grupos profesionales.

Artículo 141 de la LGSS referido a compatibilidades en el percibo de prestaciones económicas por incapacidad permanente, reflejando como profesión de referencia para la compatibilidad como la profesión para la que se tiene la incapacidad total la que ejercía el interesado o la del grupo profesional en que aquélla estaba encuadrada y siempre y cuando las funciones no coincidan con aquellas que dieron lugar a la incapacidad permanente total.

\section{VALORACIÓN PROFESIONAL DE LA INCAPACIDAD EN RELACIÓN AL CÓDIGO NACIONAL DE OCUPACIÓN. EL RD 625/2014}

Si bien hace referencia a la incapacidad temporal de menos de 365 días, introduce como un factor a tener en cuenta en el control y actualización de la situación de baja el código nacional de ocupación.

- El conocimiento del código nacional de ocupación a través de los partes médicos de incapacidad temporal que transmiten los servicios públicos de salud supondrá un avance en el conocimiento del comportamiento de esta prestación económica, que permitirá la comparación de datos a nivel internacional.

- Son datos obligatorios del parte de baja los relativos a la fecha de la baja, la contingencia causante, el código de diagnóstico, y el código nacional de ocupación del trabajador. 
- Con el fin de que las actuaciones médicas cuenten con respaldo técnico se establecen las tablas de duración óptima tipificadas por los distintos procesos patológicos susceptibles de generar incapacidades, y su incidencia en las distintas actividades laborales.

\section{CONCLUSIÓN}

Las referencias a profesión habitual son obsoletas e imprecisas. El concepto de PROFESIÓN HABITUAL debe revisarse, tanto en su valoración médico-laboral como de referencia normativa-legal de cara a la valoración de la capacidad o incapacidad laboral en el sistema de seguridad social.

Primero porque como hemos descrito es difícil de definir en la actualidad la profesión habitual por los continuos cambios en los procesos productivos, la especialización creciente, la automatización de muchos de ellos y los nuevos trabajos en el entorno telemático.

Por otra parte la tendencia, hoy en día, además precipitada por la actual crisis económica, es la precariedad en el desempeño de una "profesión" y los continuos cambios a lo largo de una vida laboral, no tanto fundamentados en la opción personal de mejora, sino por desgracia por la necesidad de trabajar en aquello que buenamente sea posible, independientemente de gustos formación o deseos personales, que condicionan una breve temporalidad en el desempeño de los trabajos.

Sería preferible referirse cuando menos a Grupos Profesionales con similares requerimientos de capacidades, parecidas condiciones de trabajo y riesgos presentes.

El propio término de profesión es confuso e impreciso pues puede que referenciemos como profesión otros términos como ocupación, puesto de trabajo, tarea o actividad, en un medio laboral cambiante muy diversificado y especializado y en continuo cambio y con aparición de profesiones nuevas o renovación de las requisitorias de las antiguas. Amén de que la rotación por múltiples trabajos es y será algo muy habitual en la población trabajadora a lo largo de su vida laboral.

Dejando atrás la profesión como un término de fácil definición y referencia al uso de antaño como cuando se hablaba de albañil o informático o abogado o carpintero, etc. Con una rotación profesional a lo largo de la vida laboral que cada vez será mayor, abandonando ese carácter a veces y antes muy común de profesión única ejercida.

La realidad diaria nos lleva a concluir la necesidad urgente de abordar un cambio en la asignación de la incapacidad más allá de la profesión habitual, y más cuando hablamos de trabajadores de menos de 55 años, en que abordar qué es su "profesión habitual» acostumbra a ser difícil y más aún, si se trata de trabajadores mucho más jóvenes.

Y ello es tan necesario como establecer protocolos que valoren las concretas, específicas y reales, capacidades requeridas por el trabajo, sus condiciones y riesgos presentes y su formación, de un trabajo concreto, más allá de unas referencias genéricas a profesiones. Lo que contacta con una labor que debiera ser realizada y actualizada en cada ocasión por los servicios de prevención y en la que no basta la elaboración de una guía genérica de profesiones y requerimientos o referenciarla de forma genérica con las ocupaciones del código nacional de ocupación, en estos dos supuestos por tener un carácter genérico e insuficiente que no específico y determinante como sería una pormenorización de tareas y capacidades convenientemente efectuada por un servicio de prevención respecto a una ocupación o trabajo determinado.

Lo que tal vez contacte con otra realidad necesaria de cambio que es la referencia incapacitante asociada a profesión habitual, en el sistema de seguridad social, que en su situación actual permite en ocasiones llevar a la controversia de otorgar la incapacidad total para una profesión $\mathrm{y}$ reubicaciones frecuentes en otra $\mathrm{u}$ otras de similares 
requerimientos lo que conlleva o una irresponsabilidad preventiva al permitir trabajos que pueden perjudicar la salud del trabajador supuestamente mermada en su condición de incapacitado o ser fruto de la necesidad por obtener un salario a cualquier precio, lo que debiera ser solucionado con otras políticas sociales, económicas y del propio sistema de seguridad social que permitieran la rehabilitación, reintegración y reinserción laboral, o por último una situación de inadecuada concesión de la incapacidad al permitir el desarrollo de otra con similares capacidades y entorno a los que tenía en la profesión para la que fue incapacitado.

\section{REFERENCIAS BIBLIOGRÁFICAS}

1. El concepto de profesión habitual como determinante para la calificación de la incapacidad permanente total. Ana Álvarez Moreno. Lexnova.es portal jurídico. Artículo extraído del libro La reforma de la Seguridad Social 2011.

2. Memento Seguridad Social 2014. Grupo Francis Lefebvre.

3. Las Prestaciones Económicas de la Seguridad Social (Régimen General y Nivel no Contributivo). Dir. Álvarez Cortés, J. C., Ed. CEESIFV, Salamanca (2008).

4. Guía de Valoración de Incapacidad Laboral para Médicos de Atención Primaria. Madrid: Escuela Nacional de Medicina del Trabajo (ENMT). Instituto de Salud Carlos III. Ministerio de Ciencia e Innovación; 2009.

5. Fundamentos médico-legales de la incapacidad laboral permanente. ISBN: 9788498361018. Manuel García Blázquez Editorial Comares.

6. Manual de la incapacidad permanente. Carpio González M. Córdoba: Editorial Almuzara, 2007.

7. Real Decreto Legislativo 1/1994, de 20 de junio, por el que se aprueba el Texto Refundido de la Ley General de la Seguridad Social (NIPO 271-14-006-5)

8. Informe sobre el Régimen vigente de Compatibilidad de las Pensiones de Incapacidad de Seguridad Social con el trabajo remunerado. Editor: CERMI.

9. Manual de Seguridad Social, Vida Soria, J.; Monereo Pérez, J. L., Molina Navarrete, C. y Quesada Segura, R.: Comares, 8. ${ }^{\mathrm{a}}$ ed., Granada, 2012.

10. Manual práctico de Medicina Legal y Administrativa para Médicos de Atención Primaria; Pérez Pineda, B., Martínez Nieto, E. y Bullejos Muñoz, M.; Ed. Comares.

11. Derecho de Seguridad Social; Almansa Pastor, J. M.; Ed. Tecnos.

12. Las Pensiones de Invalidez Permanente, Jubilación y Muerte y Supervivencia en el sistema de la Seguridad Social Española; Gala Vallejo, C.; Ed. Ministerio de Trabajo y Seguridad Social.

13. Valoración médica y jurídica de la incapacidad laboral, César Borobia ISBN: 9788497256834. Editorial La Ley.

14. La Incapacidad Laboral. Bruna Reverter, Javier. Ed. Comares 2012.

15. Incapacidades laborales y Seguridad Social $2 .^{a}$ edición, abril 2012, Antonio Barba Mora. Editorial Aranzadi. 